\title{
ELASTIC PROPERTIES OF STATISTICALLY EQUIVALENT MATERIALS WITH VARYING INDIVIDUAL GRAIN ORIENTATIONS
}

\author{
H. KIEWEL ${ }^{1}$, H. J. BUNGE ${ }^{2}$ and L. FRITSCHE \\ ${ }^{1}$ Institute of Theoretical Physics B, ${ }^{2}$ Department of Physical Metallurgy, \\ Technical University Clausthal, Germany
}

(Received 22 June 1995)

\begin{abstract}
The elastic properties of copper metal with different individual grain orientations exhibiting the same texture are determined. We simulate the real material by two different types of clusters. The first one consists of 365 cubic grains, the second cluster is an arrangement of 181 Wigner-Seitz cells of a body centred cubic (bcc)-lattice. For each type of cluster we let the local grain orientations vary. The displacement field inside these aggregates as a result of a homogeneous deformation acting on the surface of the clusters is calculated. Although the resulting local deformation field for different individual grain orientations varies strongly, the macroscopic elastic moduli are in the frame of this simulation identical for any cluster of the same type, as it has to be for statistically equivalent materials.
\end{abstract}

KEY WORDS: Cluster method, deformation field, elastic moduli, elastic anisotropy, effective elastic constants.

\section{INTRODUCTION}

In the past decades several attempts have been made to gain access to the elastic properties of polycrystalline substances. Nearly all of these methods yield no local information about the state of deformation inside the pertinent material. The methods of Voigt (1910) and Reuss (1929) base on the assumptions that throughout the whole material the strain and stress, respectively, are constant. This is in far contrast with the familiar experimental fact that the deformation varies widely between neighbouring grains. Although these two models do not include the actual strain and stress field inside the material into the calculation they yield upper and lower bounds of the macroscopic elastic constants (Hill, 1952).

The first successful method to take the local deformation field into account, i.e. the grain interaction, was developed by Kröner (Kröner, 1958; Kneer, 1964; Kneer, 1965; Morris, 1971). If the distribution of the moduli in space is perfectly disordered this method yields the exact polycrystal constants (Kröner, 1977).

To calculate explicitly the deformation field inside a polycrystalline material, two schemes have been recently developed. The methods of Kumar (1992) and Kiewel and Fritsche (1994a, 1994b) base on the simulation of the real polycrystal by a cluster of $100-1000$ grains. This aggregate is subjected to a homogeneous deformation at the 
entire surface of the cluster. The inner state of deformation is then calculated numerically. To obtain the macroscopic elastic properties, the stress-strain relation of the macroscopically homogeneous sample is exploited.

\section{METHOD}

To determine the elastic properties of polycrystalline materials we make use of the two-stage cluster method (for a detailed description see Kiewel and Fritsche (1994b)). In a first step the microscopic behaviour of the substance which is simulated by a cluster of single grains is investigated. Thereto we let a homogeneous deformation act on the surface of the cluster. The resulting displacement field $\underline{u}(\underline{r})$ inside the material is then calculated. For that purpose we apply the cluster method, which represents a scheme to determine the local deformation field under consideration of the common conditions at the grain boundaries $S_{\alpha^{\prime}, \alpha^{*}}$ :

$$
\left.\underline{u}^{(\alpha)}(\underline{r})\right|_{s_{\alpha^{\prime}, \alpha}}=\left.\underline{u}^{(\alpha)}(\underline{r})\right|_{s_{\alpha^{\prime}, \alpha}}
$$

and

$$
\left.\underline{\sigma}_{n}^{(\alpha)}(\underline{r})\right|_{s_{\alpha^{\prime}, \alpha}}=\left.\underline{\sigma}_{n}^{(\alpha)}(\underline{r})\right|_{s_{\alpha^{\prime}, \alpha}}
$$

where $\alpha$ numbers the grains, and the stress is denoted by $\underline{\sigma}_{n}$. At the entire surface $S$ of the cluster a homogeneous displacement is given (Dirichlet problem):

$$
\left.\underline{u}\right|_{s}(\underline{x})=\underline{u}_{o}(\underline{x}) \text {. }
$$

With the knowledge of the displacement field inside the whole polycrystalline material the strain tensor can be calculated, which is defined by

$$
\epsilon_{i j}(\underline{r}) \stackrel{\text { def }}{=} \frac{1}{2}\left(\frac{\partial u_{i}(\underline{r})}{\partial x_{j}}+\frac{\partial u_{j}(\underline{r})}{\partial x_{i}}\right) .
$$

Another very instructive quantity represents the relative changes of the specific volume:

$$
\frac{\delta v(\underline{r})}{v_{0}}=\operatorname{Tr}\left(\underline{\underline{\epsilon}}(\underline{r})-\underline{\underline{\epsilon}}_{0}\right),
$$

where $€_{=0}$ results from the given homogeneous displacement at the surface of the cluster.

In a second step the macroscopic elastic properties can then be calculated by forming the averages of the strain and stress tensor over the whole volume $\mathrm{V}$ of the cluster:

$$
\left(\overline{\epsilon_{i j}}\right) \stackrel{\text { def }}{=} \frac{1}{V} \int_{V}\left(\epsilon_{i j}(\underline{r})\right) d V,
$$




$$
\left(\overline{\sigma_{i j}}\right) \stackrel{\text { def }}{=} \frac{1}{V} \int_{V}\left(\sigma_{i j}(\underline{r})\right) d V \text {. }
$$

The stress-strain relation of the macroscopically homogeneous material yields then the effective elastic stiffnesses $\hat{C}_{i j k l}$ :

$$
\overline{\sigma_{i j}}=\sum_{k, l} \hat{C}_{i j k l} \overline{\epsilon_{k l}}
$$

\section{RESULTS AND DISCUSSION}

For clarity all calculations have been carried out for macroscopically isotropic copper with no orientation correlation between different grains, i.e. the ODF and MODF are constant and equal to unity. In Table 1 the utilized single crystal stiffnesses are displayed. To get an impression of the elastic anisotropy of copper we have additionally listed the anisotropies

$$
A_{G} \stackrel{\text { def }}{=} \frac{G_{m a s}}{G_{m i n}}
$$

and

$$
A_{E} \stackrel{\text { def }}{=} \frac{E_{\text {mas }}}{E_{\min }}
$$

The extrema of the shear modulus $G$ and of Young's modulus $E$ which are labeled by the indices max and $\min$ are taken over all crystal directions. The anisotropy $A_{G}$ is identical to the well-known Zener anisotropy or its reciprocal value. To value the polycrystal data calculated with the cluster method we have displayed in Table 2 the bounds of Hill (columns Reuss and Voigt) and those of Hashin and Shtrikman (1962a, 1962b) in columns HS1 and HS2. The Kröner values which hold exactly for completely

Table 1 Single crystal values for the stiffnesses $c_{i j}$ in GPa (Bradfield, 1962) and for the anisotropy of copper.

\begin{tabular}{cccccc}
\hline & $c_{l 1}$ & $c_{12}$ & $c_{44}$ & $A_{G}$ & $A_{E}$ \\
\hline $\mathrm{Cu}$ & 169.05 & 121.93 & 75.50 & 3.205 & 2.864 \\
\hline
\end{tabular}

Table 2 Theoretical bounds and Kröner values for the elastic moduli of isotropic polycrystalline copper in GPa.

\begin{tabular}{llllll}
\hline & Reuss & HS1 & Kröner & HS2 & Voigt \\
\hline B & 137.64 & 137.64 & 137.64 & 137.64 & 137.64 \\
G & 40.12 & 46.05 & 48.26 & 49.53 & 54.72 \\
E & 109.70 & 124.29 & 129.63 & 132.68 & 144.95 \\
\hline
\end{tabular}


disordered materials in space are listed in addition. As a consequence of the invariance of the bulk modulus $B$ for all directions in the case of cubic crystal symmetry all bounds for $B$ have to be identical. However, the value of the bulk modulus yields an exact criterion to test the reliability of the extensive computer code which had to be developed for realizing the cluster method.

For our investigations we use two different types of clusters. The first one consists of cubic grains (sc-cluster). The second grain shape is the Wigner-Seitz cell of a bcclattice. The construction of the clusters is realized as follows. The centre of gravity of the first grain is put into the origin of the cluster. This grain is surrounded by nearest neighbours (second shell), next nearest neighbours (third shell) and so on. Therefore the shape of the cluster is approximately spherical. The first type of cluster contains 21 , the second 13 shells. This corresponds to 365 and to 181 grains, respectively. For any type of cluster we have used four random sets of individual grain orientations.

Every cluster is subjected to a uniaxial strain acting on the entire surface of the cluster. Figure 1 shows the relative changes of the specific volume $\delta v(\underline{r}) / v_{0}$ in equidistant steps for the four sc-clusters. In all cases, the same cut is taken through the centre of the respective cluster along the plane $z=0$ containing the strain axis.
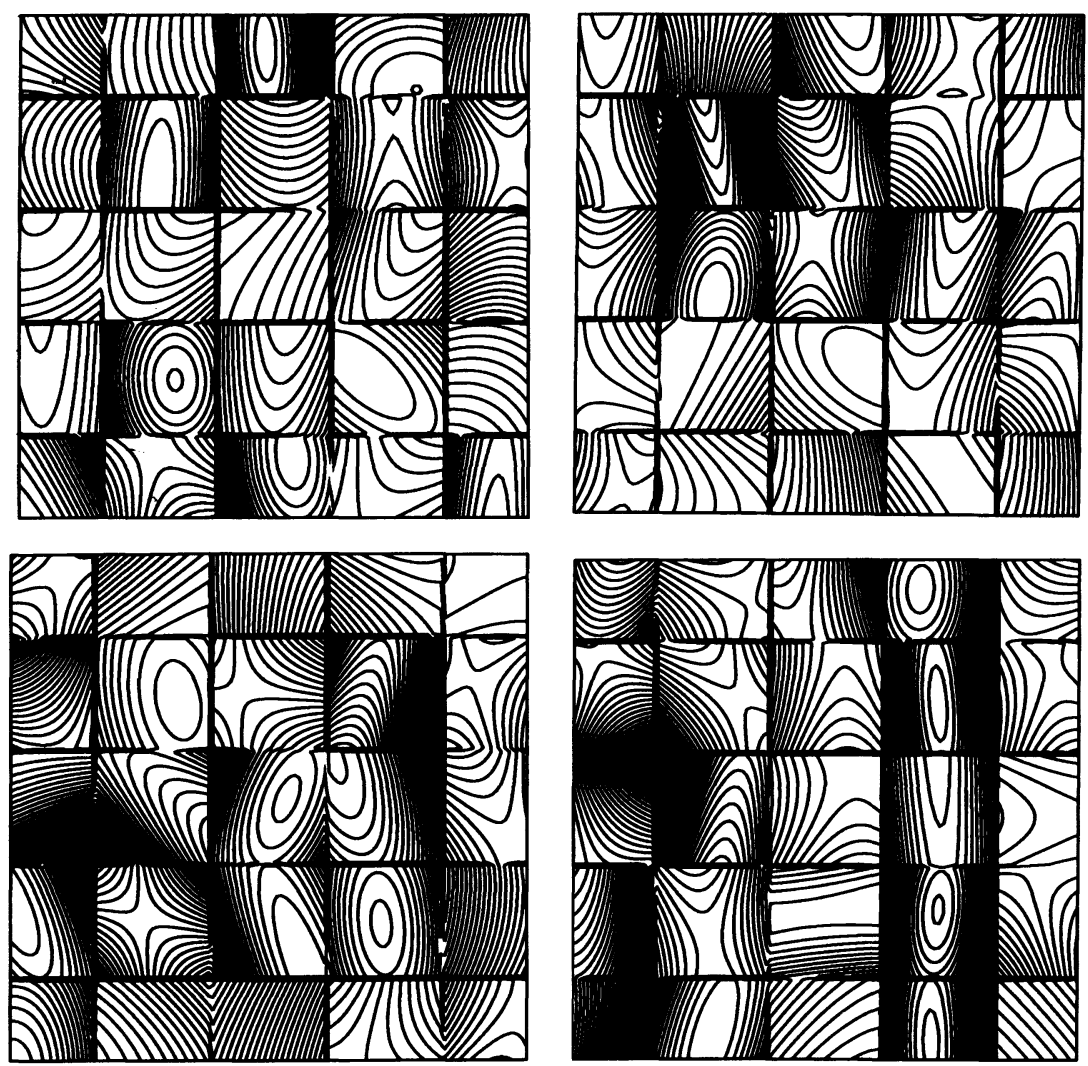

Figure 1 Relative changes of the specific volume $\delta z(\underline{r}) / v_{0}$ in equidistant steps inside four clusters consisting of cubic grains with different individual grain orientations. In all cases, the same cut is taken through the centre of the cluster $(r=\underline{0})$ along the plane $z=0$ containing the strain axis. Because of the large number of grains, we only display a narrow region centered at $\underline{r}=\underline{0}$. 
This function is discontinuous for a heterogeneous elastic medium since boundary conditions are only fulfilled for $\underline{u}(\underline{r})$ and $\underline{\sigma}_{n}(\underline{r})$ (Eqs. (1) and (2)). Therefore the arrangement of grains appears very clearly because at the grain boundaries several equidistant lines fall together. For the four clusters the local deformation is completely different. The displacement inside the clusters is, in the range of the applied displaying technique, continuous (Figure 2). A resolution of single grain boundaries seems to be impossible. Thus the boundary condition (1) is quite well fulfilled.

Figure 3 and 4 show the same fields for the bcc-cluster. As for the sc-cluster the strain tensor has to be discontinuous, in contrast to the displacement. The arrangement of grains is clearly recognizable in Figure 3. There is also a distinct local difference of the deformation field for varying individual grain orientations.

After examining the local elastic behaviour we can now determine the macroscopic elastic properties. With the aid of Eqs. (6), (7) and (8) we have calculated the elastic moduli $B, G$ and $E$ for the polycrystalline aggregates of copper (Tables 3 and 4 ). All moduli lie within the bounds of Hill and, except for one sc-cluster, all constants lie within the narrower bounds of Hashin-Shtrikman. As it has to be the values for the bulk modulus are all identical. For each type of cluster there is only a very small spread
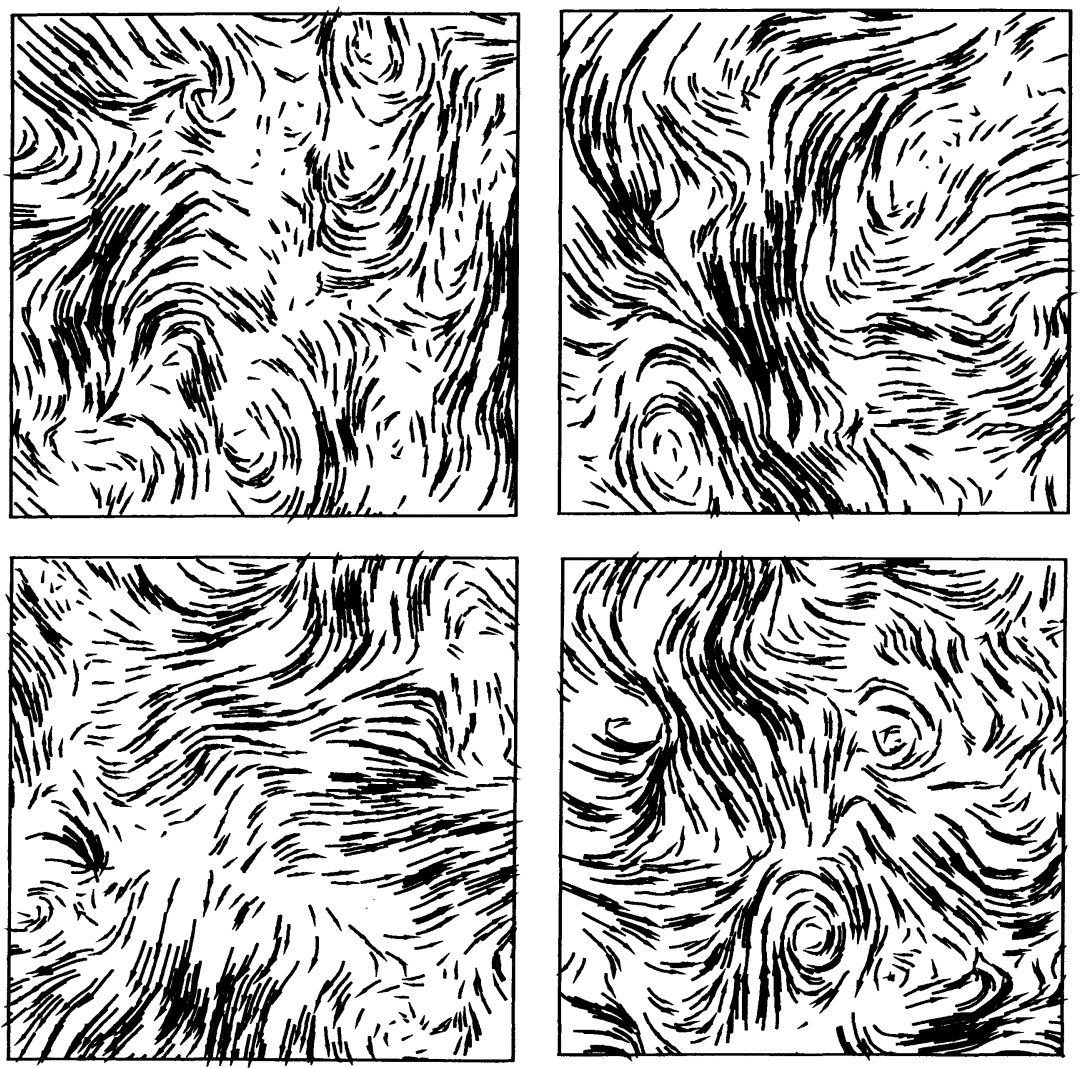

Figure 2 The displacement field $\underline{u}(\underline{r})-\underline{u}_{0}(\underline{r})$ associated with the elastically deformed clusters shown in Figure 1. 

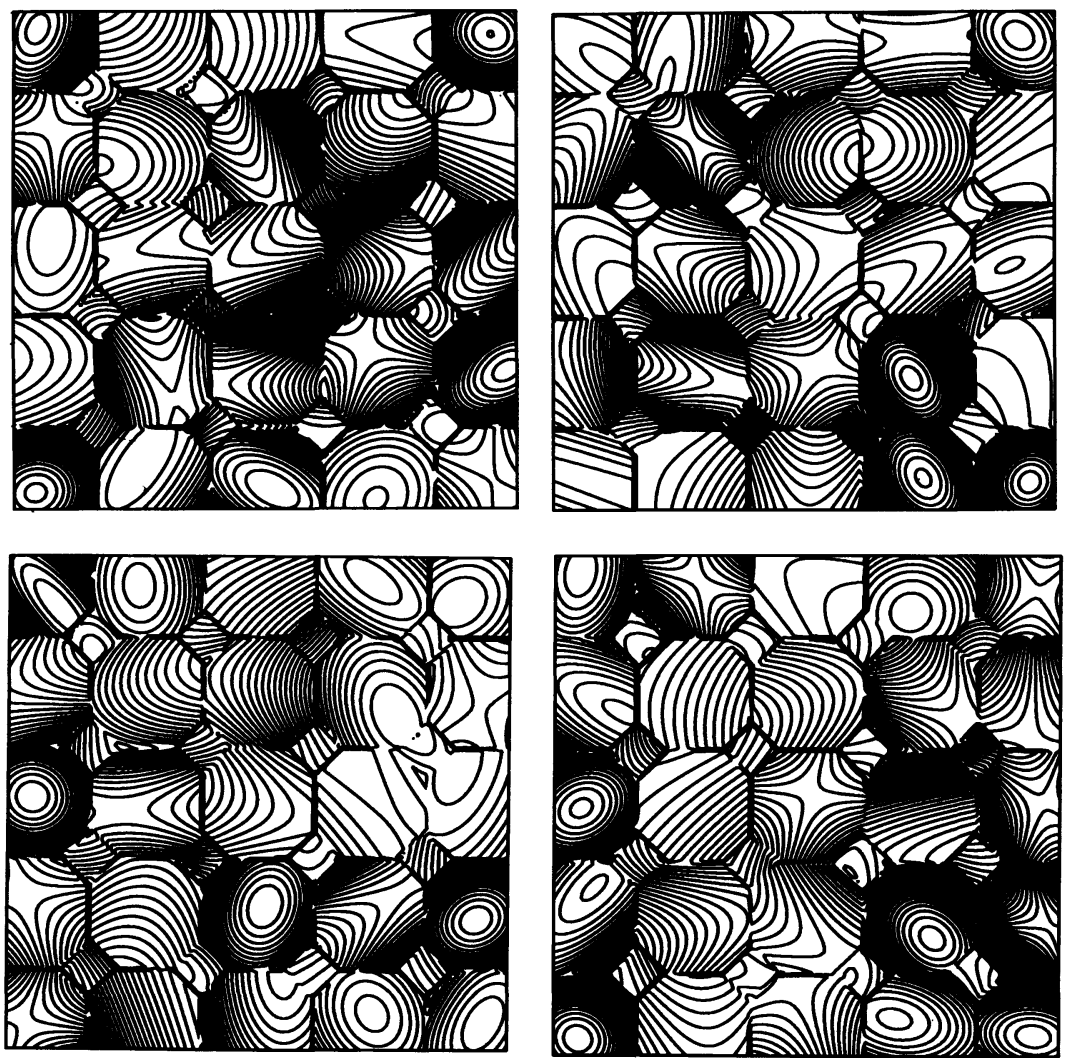

Figure 3 The function $\delta v(\underline{r}) / v_{0}$ for bcc-clusters. The situation is identical with that in Figure 1 except that the cubical grains have been replaced by Wigner-Seitz cells of a bcc-lattice.

Table 3 Elastic moduli (in GPa) and anisotropy calculated for different local grain orientations. The letters (a) to (d) stand for the respective clusters in Figures 1 and 2 consisting of cubes. $\phi$ denotes the arithmetic mean of the four cluster values.

\begin{tabular}{cccccc}
\hline & $B$ & $G$ & $E$ & $A_{G}$ & $A_{E}$ \\
\hline (a) & 137.64 & 49.34 & 132.22 & 1.056 & 1.061 \\
(b) & 137.64 & 49.36 & 132.27 & 1.088 & 1.087 \\
(c) & 137.64 & 49.49 & 132.58 & 1.031 & 1.053 \\
(d) & 137.64 & 49.62 & 132.89 & 1.031 & 1.051 \\
$\phi$ & 137.64 & 49.45 & 132.49 & 1.052 & 1.063 \\
\hline
\end{tabular}

in the values of $G$ and $E$ for the various local orientations in contrast to the enormous local differences in the deformation field displayed in Figures 1 to 4 . Because of the finite number of grains, the sample symmetry is only approximately isotropic, i.e. $A_{G} \approx 1$ and $A_{E} \approx 1$. There is a noticeable difference for the shear and Young's modulus between the two types of cluster, which has to be interpreted as an effect of the various grain shapes. 

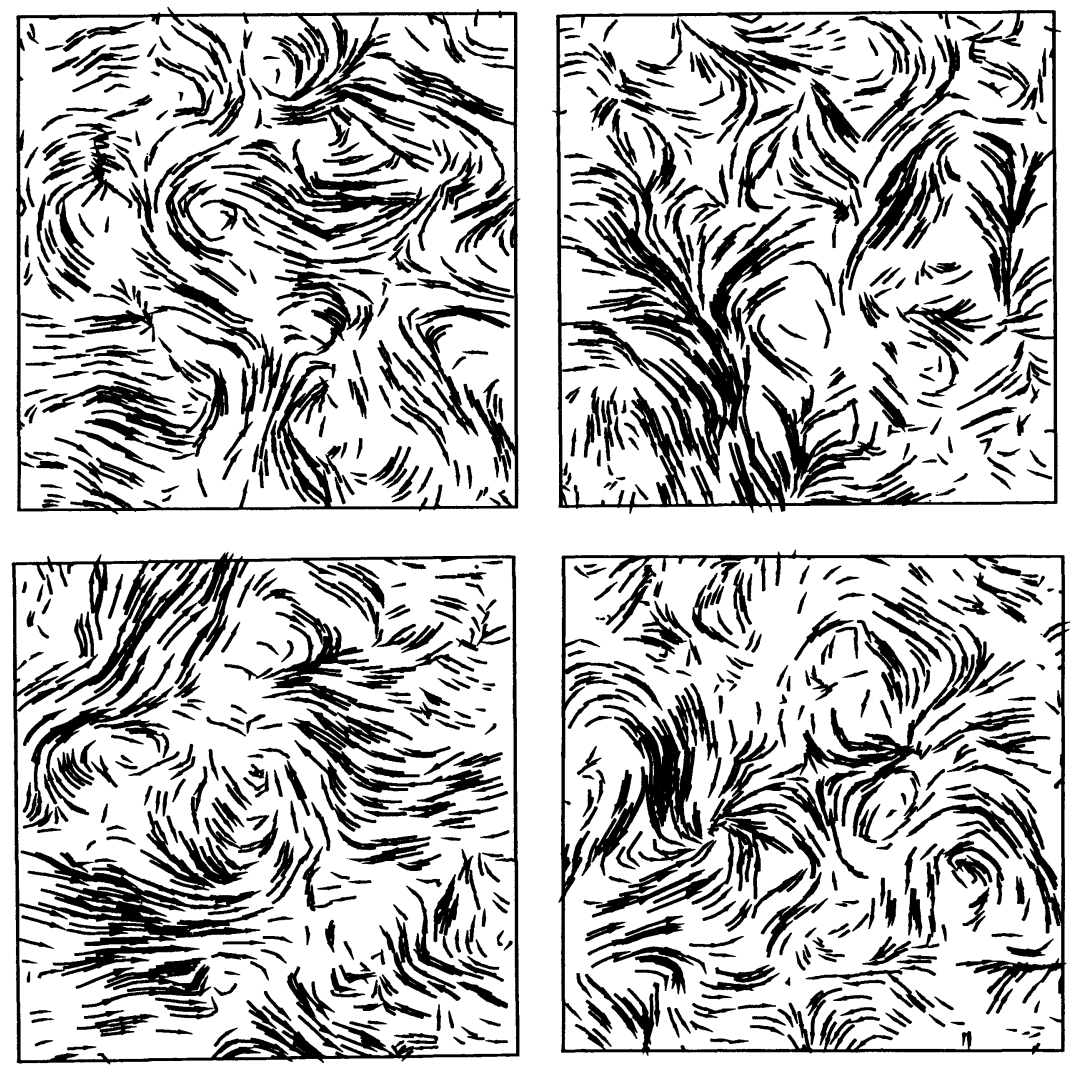

Figure 4 The displacement field $\underline{u}(\underline{r})-\underline{u}_{0}(\underline{r})$ associated with the elastically deformed clusters shown in Figure 3.

Table 4 Elastic moduli (in GPa) and anisotropy calculated for the respective clusters (a) to (d) in Figures 3 and 4 consisting of Wigner-Seitz cells of a bcc-lattice. $\phi$ denotes the arithmetic mean of the four cluster values.

\begin{tabular}{lccccc}
\hline & $B$ & $G$ & $E$ & $A_{G}$ & $A_{E}$ \\
\hline (a) & 137.64 & 47.55 & 127.92 & 1.061 & 1.069 \\
(b) & 137.64 & 47.46 & 127.70 & 1.071 & 1.079 \\
(c) & 137.64 & 47.43 & 127.63 & 1.063 & 1.072 \\
(d) & 137.64 & 47.66 & 128.18 & 1.078 & 1.094 \\
$\phi$ & 137.64 & 47.53 & 127.86 & 1.068 & 1.079 \\
\hline
\end{tabular}

\section{CONCLUSIONS}

The investigations of the present paper show that the reliability of the cluster method holds not only for the determination of the macroscopic elastic moduli but also for the calculation of the local deformation field inside polycrystalline materials. For elastically anisotropic grains the local deformation field is completely different for 
various individual grain orientations. In spite of this strong local variance the macroscopic properties are, in the range of the employed accuracy, identical for statistically equivalent samples. As it has to be, the macroscopic quantities of a material are uniquely defined by its full set of statistical parameters.

\section{Acknowledgements}

The present work was financially supported by the Deutsche Forschungsgemeinschaft which is gratefully acknowledged.

\section{References}

Bradfield, G. (1962) Private communication to Kneer, G. (quoted in (Kneer, 1965)).

Hashin, Z. and Shtrikman, S. (1962a) On some variational principles in anisotropic and nonhomogeneous elasticity, J. Mech. Phys. Solids 10, 335.

Hashin, Z. and Shtrikman, S. (1962b) A variational approach to the theory of the elastic behaviour of polycrystals, J. Mech. Phys. Solids 10, 343.

Hill, R. (1952) The elastic behaviour of a crystalline aggregate, Proc. Phys. Soc. A 65, 351.

Kiewel, H. and Fritsche, L. (1994a) Calculation of average elastic moduli of polycrystalline materials including $\mathrm{BaTiO}_{3}$ and high- $\mathrm{T}_{\mathrm{c}}$ superconductors, Proc. ICOTOM-10, Materials Science Forum 157-162, 1609.

Kiewel, H. and Fritsche, L. (1994b) Calculation of effective elastic moduli of polycrystalline materials including non textured samples and fiber textures, Phys. Rev. B 50, 5.

Kneer, G. (1964) Zur Elastizität vielkristalliner Aggregate mit und ohne Textur, Doctoral Thesis, Technische Universität Clausthal.

Kneer, G. (1965) Über die Berechnung der Elastizitätsmoduln vielkristalliner Aggregate mit Textur, phys. stat. sol. 9, 825 .

Kröner, E. (1958) Berechnung der elastischen Konstanten des Vielkristalls aus den Konstanten der Einkristalle, Z. Phys. 151, 504.

Kröner, E. (1977) Bounds for effective elastic moduli of disordered materials, J. Mech. Phys. Solids 25, 137.

Kumar, S. (1992) Computer simulation of 3D material microstructure and its application in the determination of mechanical behavior of polycrystalline materials and engineering structures, $\mathrm{Ph}$. D. Thesis, Pennsylvania State University.

Morris, P.R. (1971) Iterative scheme for calculating polycrystal elastic constants, Int. J. Eng. Sci. 9, 917.

Reuss, A. (1929) Berechnung der Fließgrenze von Mischkristallen auf Grund der Plastizitätsbedingung für Einkristalle, Z. Angew. Math. Mech. 9, 49.

Voigt, W. (1910) Lehrbuch der kristallphysik, Teubner, Berlin. 